\title{
AS: Automated Agriculture System based on WSN
}

\author{
Seong-eun Yoo, Jae-eon Kim, Taehong Kim, Sungjin Ahn, Jongwoo Sung, Daeyoung Kim \\ Real-time Embedded Systems Laboratory, Information and Communications University \\ \{seyoo, jekim, damiano, sungjin, jwsung, kimd@icu.ac.kr\}
}

\begin{abstract}
This paper describes the results of real deployment of $A^{2} S$ which consists of WSN(Wireless Sensor Network) to monitor and control the environments and a management sub-system to manage the WSN and provide various and convenient services to consumers with hand-held devices such as a PDA living a farming village. The WSN were deployed in greenhouses with melon and cabbage in Dongbu Handong Seed Research Center. $A^{2} S$ was used to monitor the growing process of them and control the environment of the greenhouses. We acquired valuable experiences and ideas from this real deployment and operation of $A^{2} S$ and believe that they can be useful in consumer electronics field such as home network as well as automated agriculture field. .
\end{abstract}

\section{Introduction}

In recent years, the desire to connect all electronic computing devices together has increased. Although they can be connected through wired lines, it is more convenient and effective to use wireless links when we consider the large number of pervasive devices in the environment. In the coming years, it is expected that the Wireless Sensor Network(WSN) will be used commonly in applications in consumer electronics, PC peripherals, home automation, home security, personal healthcare, toys and games, industrial control and monitoring, asset and inventory tracking, intelligent agriculture, and so on [5] [10]. Most of developments and experimental deployments of WSN are inclined to be achieved for citizen in towns. However, there're some researches to share the technology with people in a farming village [1] [2] [3]. [1] reported the result of deployment in a vineyard. Considering placing the sensor nodes with fairly high precision, the researchers used a planned network rather than a network with ad-hoc routing. For reliability, they provided route diversity and multiple transmissions. The authors demonstrated some of the value that a wireless sensor network might deliver to an agricultural setting. In addition to demonstrating the total cost of ownership of a

This research was supported by the MIC(Ministry of Information and Communication), Korea, under the ITRC(Information Technology Research Center) support program supervised by the IITA(Institute of Information Technology Advancement)" (IITA-2006-(C1090-06030047)) wireless network is lower than a wired network, they could also show one example, frost prevention where a live network is more valuable than a standard data logger. [2] [3] presented Lofar Agro project that concentrated on monitoring micro-climates in a crop field. The pilot project concerned the protection of a potato crop against phytophthora, a fungal disease that can spread easily among plants and destroy a complete harvest within a large region. The authors described a precision agriculture architecture based on WSN. They deployed a large-scale sensor network (about 100 nodes) and acquired valuable experiences. These papers are very useful references for WSN applications in the intelligent agriculture field. However, they have a little bit weak points. They can gather the environmental information, but they are weak to control the environment of the field.

The contributions of our paper are summarized as follows. The system architecture for automated agriculture system is presented from sensor/actuator node hardware in the bottom to management sub-system in the top and is evaluated in the real-deployment. The way to control the environment using the feedback from the gathered information is described in the paper. The paper gives the experiences of real deployment in greenhouses and the ideas to improve the system.

The rest of this paper is organized as follows: Section 2 presents the system architecture of $A^{2} S$ and then Section 3 describes the deployment result and discussion for the result. Finally, the paper concludes with a summary of our work and a statement of future work in Section 4 .

\section{System Architecture}

$\mathrm{A}^{2} \mathrm{~S}$ consists of WSN, gateways, and a management sub-system. 25 sensor nodes, 1 actuator node, and 3 sink nodes are deployed in greenhouses and operated during severely cold last winter and one of the sensor nodes is plunged to a field near to one of greenhouses in order to endure very harsh condition of heavy snows and coldness of $-15^{\circ} \mathrm{C}$ [11]. 3 industrial PC-based gateways are installed to transform data over RS-232 from sink nodes to data over TCP/IP to servers. WLAN APs with directional antennas provide the long range wireless link between WSN and the management sub-system which is about $0.5 \mathrm{~km}$ far a way from greenhouses. The management subsystem with a DB-server and a web- 
server manage WSN and provide easy interface to farmers with hand-help devices such as a PDA.

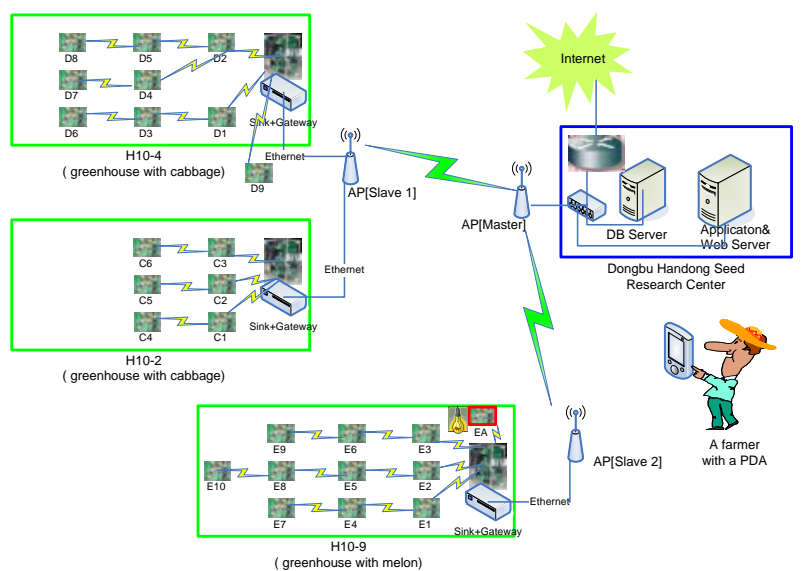

Figure 1. Overall System architecture

\subsection{Agricultural WSN}

A-node is an Agriculture sensor node developed to be deployed in a greenhouse and to sense the environment of the greenhouse. The node is embedding all of 8bit MCU, IEEE 802.15.4 compatible transceiver (in $2.4 \mathrm{GHz}$ band), a CPLD(sleep timer to wake up the MCU from power down mode) and sensors(ambient light sensor, temperature and humidity sensor) in one PCB to reduce possibilities of defects. A sensor node is equipped with a battery of lithium-ion rechargeable cells and monitors the voltage level of battery for maintenance purpose. To prevent A-node from the humidity in the greenhouse, protection paint is used. The software of A-node is based on the initial version of ANTS-EOS [4]. EOS is a lightweight C-based multi-threaded Operating System which is developed to supports multiple sensor network platforms. The network protocols of EOS are customized for this project to a light-weight CSMA based MAC and a robust multi-hop ad-hoc routing protocol, which are described at the end of this sub-section. After setting up the network topology, A-node runs its application software. The application software begins its active period by turning on its sensors and sensing the environment of the greenhouse. The application software reads temperature, humidity, and luminance of a greenhouse from sensors and reports the result to the sink via its parents. If it receives any packets from its children during this active time, it relays the packets to its parent. After the transmission of its sensing data, A-node waits for its working schedule such as sensing period in sleep order message from the sink. As it receives its sensing period, the application software turns off its attached sensors and puts the transceiver to power down mode. Finally, it sets up the internal sleep timer(the external sleep timer is not used because of its high current consumption), goes to its sleep period and waits for the expiration of the timer. After the expiration of the timer, the application restarts its next active periods by turning on the transceiver and the sensors and continues to sense the environment of the greenhouse.

$C$-node(aCtuator node) is designed to control the intensity of illumination of the greenhouse with growing melon. It has an additional relay board to A-node to control the switch of the lights in the greenhouse. It's mainly powered and doesn't use any power saving scheme. The application software of $C$-node waits for the command from the sink. Whenever it receives the command from the application server via the sink, it controls its relay to turn on or off the lights.

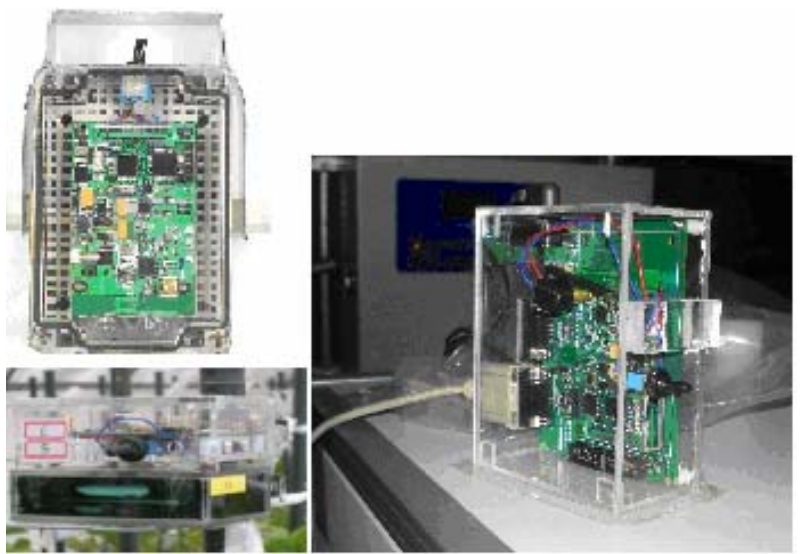

Figure 2. $A$-node $\left(1^{\text {st }}\right.$ version: lower-left, $2^{\text {nd }}$ version: upper-left) and a sink node(right)

The sink node is developed to gather the sensing information from A-nodes and transmit commands to Anode and $C$-node. As the core component of the gateway, the sink node has an additional interface board to A-nodes to provide RS-232 serial link to the gateway. The main MCU module has the same hardware specification with A-node except that the sink node is not equipped with any sensors. The sink node keeps the sensing schedule from the application server and schedules the operation of the internal A-nodes by sending sleep order message every sensing period. The order-based sleep scheme to conserve the current consumption using the sleep order message is described in detail at the end of this subsection.

A light-weight CSMA based MAC protocol and a robust multi-hop ad-hoc routing protocol are implemented. When A-node has a packet to send, it checks CCA. If the channel is idle, then the packet is transmitted. If there is no acknowledgement from the recipient of the packet, MAC layer retransmits the packet up to 3 times. To simplify the route discovery, a tree-level which is the same as hop count from the sink node is preprogrammed in on-chip EEPROM with a network-wide unique 16bit 
address. The level of the sink node is ' 0 ', level ' 1 ' is assigned to the children of the sink node, level ' 2 ' ' is assigned to the grandchildren of the sink node, and so on. When A-node initializes its parent-child relationship, it begins the joining process by broadcasting the 'search parent' packet including a cost function, in this case its tree-level. Whenever a sensor node receives the 'search parent' packet, it checks the cost function and unicasts the 'invite' packet to the joining node if the received treelevel is one-level larger than it own tree-level. For the specified time, the joining node waits for the 'invite' packets from the prospective parent nodes and stores the RSSI values with the 'invite' packets. After the specified time, the joining node chooses its parent according to the cost function, in this case the largest RSSI value, and sends the 'join' packet to its parent. The sensor node which receives the 'join' packet adds the joining node to its children list. Finally, the joining process is finished and the parent-child relationship is established. Whenever the parent of an A-node is considered to be dead, the $A$ node initiates the joining process by trying to search a new parent with immediate lower tree-level as quickly as possible.
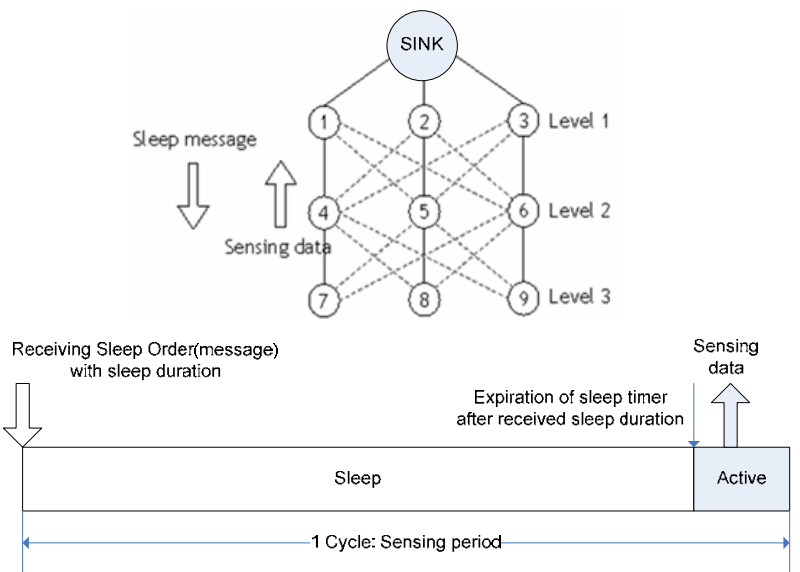

Figure 3. order-based sleep scheme

To conserve power, order-based sleep scheme is used. Whenever the sensing schedule( sensing period ) is set (or ordered) by the application server, the sink node keeps the schedule and it spreads the sleep order message over its network every sensing period. Whenever A-node receives the sleep order message, it sets the expiration time of its sleep timer to the value of the duration field included in the message. When the timer is expired, $A$ node senses its ambient environment and the voltage level of its battery and sends the data to the sink and waits for the next sleep order message. To use this power saving scheme, it is required that the parent node of a node awake earlier than the node in order to relay the sensing data of the child node. Since the sleep order message is spread out from the sink level-by-level, the requirement is met.

Rather than placing A-node randomly, we placed each A-node at the priori planned positions which had been decided by agriculturists considering the cultivation. The initial sensing period is set to the minimum, 20 seconds to test the operation of A-nodes quickly and then the sensing period is settled to 5 minutes. After running from the gateway to the application server, A-nodes are placed one bye one from the vicinity of the sink. Figure 4 shows the pictures of A-nodes which are deployed in the greenhouses and the outside.

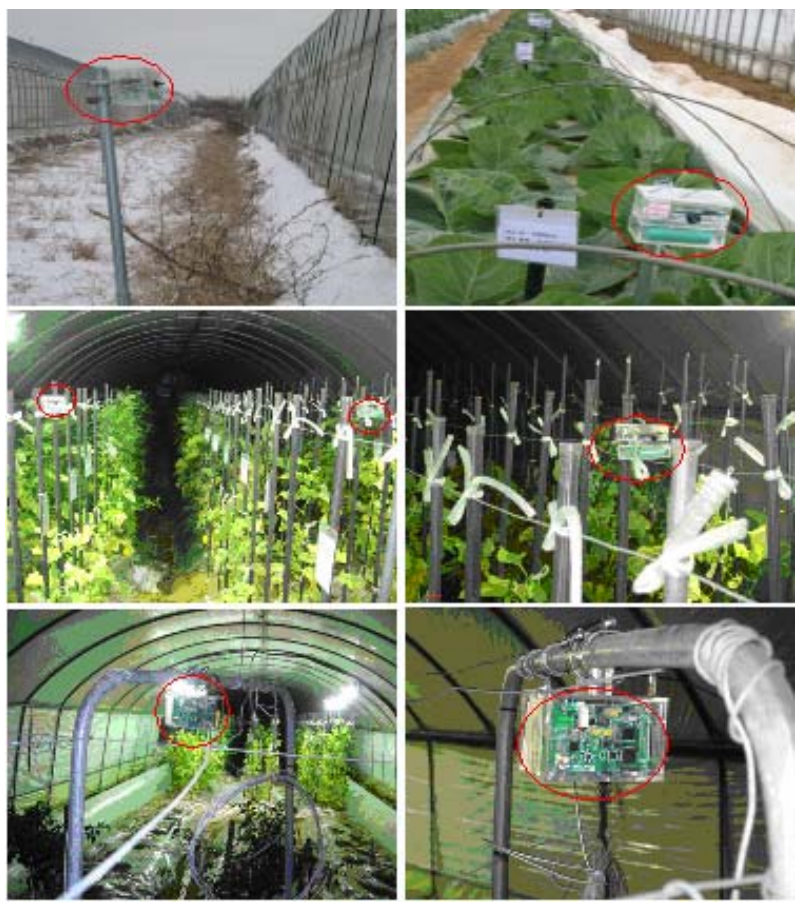

Figure 4. A-node(top-leftmost) in the outside of the greenhouse. A-nodes(red-circled ones) deployed in greenhouses with cabbage(top-leftmost) and melon

\subsection{Gateway \& Management sub-system}

The gateway transforms data over RS-232 from a sink to data over TCP/IP to server and vise versa. The gateway is implemented on a Pentium-M $1.6 \mathrm{GHz}$ based industrial PC. The gateway is connected to AP via a WLAN link or a wired ethernet link. The AP is connected to the management sub-system in the distance of $0.5 \mathrm{~km}$ via WLAN link.

The management sub-system consists of a DB server, an application server, and a web-server. The application server receives data from WSN and stores them in the DB server and provides the way to configure the WSNs. The schedule of sensing is configured by the application 
server. The whole sensing data are stored in the DB server and can be accessed by users(PC or PDA) via the web-server.

\section{Result and discussions}

$\mathrm{A}^{2} \mathrm{~S}$ was operated 1-month in severely cold winter. From this real deployment, we acquired the following lessons.

LOS(Line Of Sight) communication range of A-node was up to $70 \mathrm{~m}$. However, the range was reduced about $30 \mathrm{~m}$ in greenhouse because of many interfering sources such as iron wires and foliage (Figure 5). Considering the stable RF link and the sensing range, we limited the separation up to $20 \mathrm{~m}$.

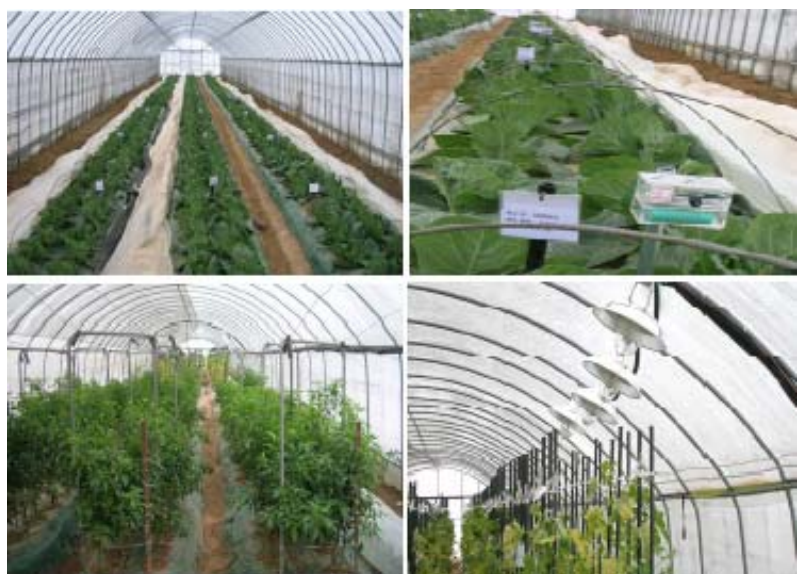

Figure 5 A-nodes among many $\mathrm{RF}$ interferers such as iron wires and foliage.

Originally, A-node was planned to be waked up from the sleep mode by an external timer in on-board CPLD to reduce the current drawing. However, the power consumption of the CPLD was measured relatively high and was useless. We lost some of sensing data because the battery exhaustions of some sensor nodes. It is recommended to use a real-time clock using an internal timer and an external low frequency crystal in order to minimize the power consumption.

Although the order-based sleep scheme has a strong point that it doesn't require any complex 'time synchronization' schemes, the parent node should be awoken earlier than the child node to relay the sensing data of the child node. As explained in the previous section, because the sleep order message is spread out from sink to nodes level-by-level, the parent node goes to sleep mode earlier than the child node, the parent node awakes earlier than the child node.

Although we used very accurate sensors, they did not show the same output levels in the same place(Figure 6). We found that there were interferences from some components such as power-control ICs in the PCB and some components such as batteries in the same enclosure. It is recommended for the sensors to be isolated from the other interfering components in the PCB and the enclosure. The Figure 7 shows the comparison between KMA(Korean Meterological Administration) standard and WSN measured. The difference is up to $4.5^{\circ} \mathrm{C}$ and average of $2.7^{\circ} \mathrm{C}$. By calibrating the sensors, the sensing result is expected to be more accurate.

Since A-node was planned to be operated during 1 month for field test, it wasn't equipped with any power generator. A solar recharging circuit may be used to recharge the rechargeable battery.

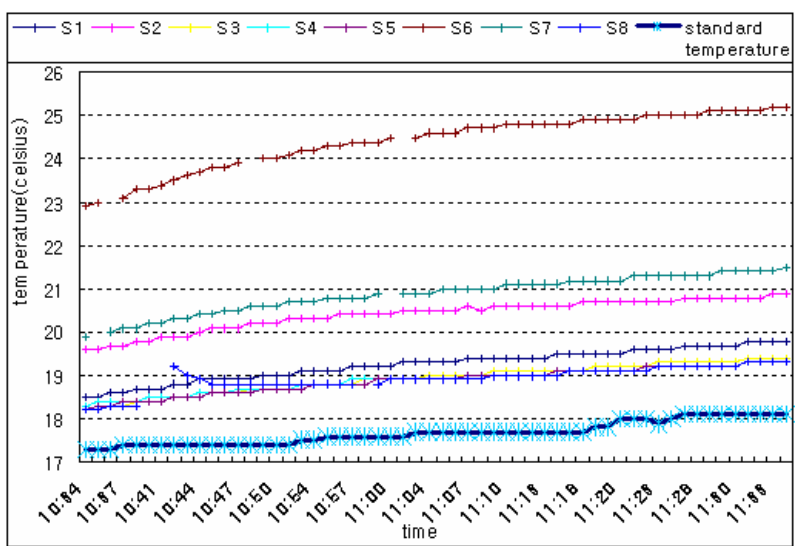

Figure 6. variations of measured temperature(the experimental data from Dongbu IT Inc.)

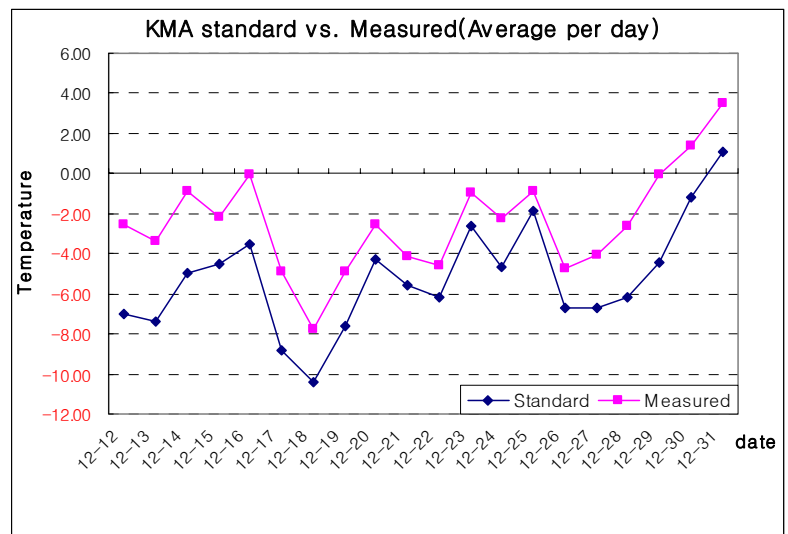

Figure 7. KMA standard vs. WSN measured(the experimental data from Dongbu IT Inc.)

\section{Conclusion}

In this paper, we report the results of real-deployment of $\mathrm{A}^{2} \mathrm{~S}$ which is designed and implemented to realize automated agriculture. From the sensor node hardware to the management system, the whole system architecture is explained. For low power consumption, we propose the 
order-based sleep scheme. Especially, the experiences and lessons which we acquired from the real deployment are discussed.

On the future work, we are studying on more stable low power scheduling communication scheme and developing more miniaturized agricultural sensor node. We are under developing WSN for an apple orchard with SNR Inc [12].

\section{Acknowledgment}

All RESL(Real-time Embedded System Lab.) members were not liberal to give advices and their times. We appreciate their valuable help. We're grateful to Kihyun Kim and Mahn-cheol Shin in Sysmate Inc. for their valuable help in agricultural sensor nodes hardware development. We're grateful to Inmook Na, Seok-cheol Chang, Youngju Hwang, and Sung-il Ham of Dongbu IT CO., LTD., Jaeheung Lee of Dongbu Handong Seed Research Center, and Ki-po Yun of UCT Inc. for their experimental data and advices.

\section{References}

[1] R. Beckwith. D. Teibel, and P. Bowen Jones, "Report from the Field: Results from an Agricultrual Wireless Sensor Network", proceedings of $29^{\text {th }}$ IEEE LCN'04, Tampa, Florida, November 15-17, 2004

[2] A. Baggio, "Wireless sensor networks in precision agriculture”, proceedings of REALWSN'05, Stockholm, Jun 20-21, 2005

[3] K. Langendoen, A. Baggio, O. Visser, "Murphy Loves Potatoes: Experiences from a Pilot Sensor Network Deployment in Precision Agriculture", 14 ${ }^{\text {th }} 14$ th Int.
Workshop on Parallel and Distributed Real-Time Systems (WPDRTS), Rhodes, Greece, April, 2006

[4] D. Kim, T. Sanchez Lopez, S. Yoo, J. Sung, J. Kim, Y. Kim, Y. Doh, “ANTS: An Evolvable Network of Tiny Sensors”, Lecture Notes in Computer Science(LNCS vol. 3824), pp. 142-151, 2005

[5] Jose A. Gutierrez, Ed. Callaway, and Raymond. Barrett, "Low-Rate Wireless Personal Area Networks: enabling wireless sensors with IEEE 802.15.4”, IEEE Press, 2004

[6] Masayuki Hirafuji, et al, “Advanced Sensor-Network with Field Monitoring Servers and MetBroker”, CIGR International Conference, Beijing 2004, No. 30-125A

[7] T. Fukatsu, et al, "Field monitoring using sensornodes with a web server", Journal of Robotics and Mechatronics, 2005. 17(2):164-172.

[8] Dongxian He, et al, "A Crop Field Remote Monitoring System Based on Web-server-embedded Technology and CDMA Service”, 2007 International Symposium on Applications and the Internet Workshops (SAINTW'07)

[9] Field Server, http://model.job.affrc.go.jp/FieldServer / FieldServerEn/default.htm

[10] http://www.zigbee.org

[11] http://www.kma.go.kr, Korea Meteorological Administration

[12] http://www.snre.co.kr 